\title{
Instalação de implante imediato em alvéolo fresco associado a enxerto com substituto ósseo
}

\author{
Immediate implant in fresh socket associated with bone \\ substitute graft
}

\section{Implante inmediato en cavidad fresca asociada con injerto sustituto óseo}

\author{
Sabrina Oliveira Prado ${ }^{1}$ \\ Lucas Monteiro de Vasconcelos Alves de Souza ${ }^{2}$ \\ 1 sabrina_oliveira02@hotmail.com \\ 2 lucas.monteiro.souza.usp@gmail.com \\ Endereço para correspondência: \\ Lucas Monteiro de Vasconcelos Alves de Souza \\ SMAS, Lote 1, Trecho C, Bloco C, Apto. 501 \\ 71218-010 - Brasília - Distrito Federal - Brasil \\ E-mail: lucas.monteiro.souza.usp@gmail.com
}

Recebido: 22.04 .2020

Modificado: 20.07.2020

Aceito: 21.08.2020

\begin{abstract}
RESUMO
A perda dentária é causadora de perda do volume óssea do rebordo dos maxilares e pode inviabilizar a instalação de implantes dentários e provocar defeitos estéticos importantes em regiões anteriores. Para evitar estes defeitos indesejáveis, a odontologia desenvolveu diversas técnicas com objetivo de preservar a arquitetura periodontal óssea e gengival. Dentre estas técnicas está a instalação de implantes imediatos em alvéolos frescos associado a preenchimento do gap vestibular com substitutos ósseos. Para alcançar as altas taxas de sucesso que a literatura expõe é necessário que se respeite os princípios biológicos e técnicos do procedimento, espacialmente em regiões anteriores superiores que exigem maior sensibilidade operacional. $\mathrm{O}$ objetivo deste trabalho é o relato de caso de instalação de implante imediato em região estética associado a substituto ósseo e discutir os principais aspectos técnicos desta cirurgia.
\end{abstract}

PALAVRAS-CHAVE: Implantes dentários. Regeneração óssea. Osseointegração.

\footnotetext{
ABSTRACT

Tooth loss causes loss of bone volume in the jaw and may make it impossible to install dental implants and cause significant cosmetic damage to the anterior regions. To avoid these undesirable defects, dentistry develops several techniques in order to preserve the bone and gingival periodontal architecture. Among these techniques is installing immediate implants in fresh alveoli associated with filling buccal gaps with bone substitutes. To achieve the high success rates that the literature exposes, it is necessary to respect the technical and biological principles and procedures, spatially in superior anterior regions and with greater operational accuracy. The aim of this study is the case of immediate installation of the immediate implant in the aesthetic region associated with bone replacement and discuss the main technical aspects of this surgery.
} 
KEYWORDS: Dental implants. Bone regeneration. Osseointegration.

\section{RESUMEN}

La pérdida de dientes provoca la pérdida de volumen óseo en la mandíbula y puede imposibilitar la instalación de implantes dentales y causar daños cosméticos significativos en las regiones anteriores. Para evitar estos defectos indeseables, la odontología desarrolla varias técnicas para preservar la arquitectura periodontal ósea y gingival. Entre estas técnicas está la instalación de implantes inmediatos en alvéolos frescos asociados con el llenado de huecos bucales con sustitutos óseos. Para lograr las altas tasas de éxito que expone la literatura, es necesario respetar los principios y procedimientos técnicos y biológicos, espacialmente en las regiones anteriores superiores y con mayor precisión operativa. El objetivo de este estudio es el caso de la instalación inmediata del implante inmediato en la región estética asociada con el reemplazo óseo y discutir los principales aspectos técnicos de esta cirugía.

PALABRAS CLAVE: Implantes dentales. Regeneración ósea. Oseointegración. 


\section{INTRODUÇÃO}

A perda dentária é causadora de perda do volume óssea do rebordo dos maxilares e pode inviabilizar a instalação de implantes dentários e provocar defeitos estéticos importantes em regiões anteriores. Para se evitar sequelas e ausência de volume ósseo para instalação de implantes osseointegrados, a odontologia desenvolveu diversas técnicas de manutenção e reestabelecimento de ósseo alveolar. Dentre estas técnicas, a instalação de implantes imediatos é uma boa opção para reestabelecimento de dente em regiões estéticas mantendo ou devolvendo o arcabouço ósseo do rebordo dentário ${ }^{1}$.

A exodontia, precedida da instalação de implantes imediatos, deve ser realizada com cautela, de modo atraumático aos tecidos periodontais, preservando paredes ósseas do alvéolo e arquitetura gengival. Além disso, a instalação dos implantes imediatos também deve ser realizada com atenção, pois apenas o bom posicionamento destes proporcionará a preservação da quantidade e qualidade dos tecidos peri-implantares. Outro aspecto importante são os desenhos e conexões protéticas dos implantes, que quando bem escolhidos terão boa interação e compatibilidade com os tecidos promovendo saúde e estética gengival ${ }^{2}$.

A instalação de implantes imediatos permite o aumento de tecido duro, por meio do preenchimento de gaps, e de tecido mole, por meio de enxertos gengivais, durante o procedimento, devolvendo tecidos perdidos e mantendo tecido hígidos. Deste modo, em um único procedimento, o paciente pode receber o aparato necessário para a instalação de prótese sobre implante ${ }^{3}$.

O sucesso da instalação dos implantes imediatos depende de numerosos fatores intrínsicos e extrínsicos. Os fatores intrínsecos são dependentes do paciente e incluem a relação entre tecidos duros e moles, fenótipo gengival e posição da raiz sagital no osso alveolar. Os fatores extrínsecos, por outro lado, dependem do clínico e incluem a posição e a angulação tridimensionais do implante, além da seleção do contorno do pilar e da restauração provisória ${ }^{4-5}$.

A instalação de implantes imediatos, realizados no mesmo momento que a exodontia, por reduzir a quantidade de intervenções cirúrgicas e preservarem os tecidos periimplantares, é muitas vezes a melhor escolha no plano de tratamento reabilitador. A literatura tem embasado o cirurgião na seleção desta técnica, visto que a taxa de sucesso dos implantes imediatos é similar a dos implantes instalados após a cicatrização óssea ${ }^{6-7}$.

Um estudo de meta-análise comparando os índices de sucesso dos implantes imediatos com os que seguiram o protocolo clássico de 2 estágios. Segundo os autores, existe risco maior de implantes imediatos falharem em comparação aos implantes tardios, visto que é composto por etapas cirúrgicas mais sensíveis à técnica do operador ${ }^{8}$.

No entanto, a literatura mostra que a taxa de sobrevida de implantes imediatos é similar à de implantes tardios. De acordo com revisão sistemática, os implantes imediatos apresentam taxa de sobrevida de $97.44 \%$, enquanto que implantes tardios, realizados em rebordo cicatrizados, apresentam $96.23 \%$ de taxa de sobrevida. Isso mostra que independente do estágio cirúrgico da instalação dos implantes, imediato ou tardio, a sobrevida destes será alta, caso o clínico respeite os princípios biológicos de cada técnica?

Em meta-análise na qual 16 estudos clínicos randomizados foram incluídos, foi observado um risco aumentado de falha de $3 \%$ nos implantes imediatos quando comparados aos implantes tardios. No entanto, o trabalho não apresentou diferença estatística significante no sucesso e na quantidade de reabsorção da crista óssea entre implantes imediatos e tardios. Outra observação bastante pertinente deste trabalho são os melhores resultados obtidos com implantes imediatos em regiões anteriores, enquanto que os implantes tardios resultam melhor em regiões posteriores ${ }^{10}$.

Outros artigos de revisão sistemática e meta-análise corroboram que não há diferença estatística significante na perda de tecido mole e duro marginal entre implantes imediatos e tardios. Isso significa que além da taxa de sucesso entre as técnicas ser semelhante, a longevidade e manutenção dos tecidos peri-implantares também é similar ${ }^{11-12}$.

Apesar da alta previsibilidade que os implantes imediatos apresentam em relação aos implantes instalados em rebordo cicatrizado, há a necessidade de atenção e cautela durante sua atuação, visto que alguns trabalhos relatam maior risco de falha nos implantes instalados em alvéolo fresco devido ao maior número de etapas sensíveis à erros operacionais ${ }^{13-14}$.

É importante salientar que, quando a instalação de implantes imediatos é citada como mantenedora das estruturas periodontais, a simples presença do parafuso de titânio não é capaz de evitar a reabsorção de tecidos duros e moles. A manutenção do arcabouço periodontal depende de vários outros fatores como o fenótipo gengival, anatomia do alvéolo fresco, ausência de retalhos, confecção de provisórios imediatos, biossegurança durante procedimento e no pós-operatório, entre outros ${ }^{15}$.

Além disso, técnicas de regeneração óssea guiada são muito bem-vindas em cirurgias de implantes instalados em alvéolo fresco. O uso de substitutos óssea de longa reabsorção associados a membranas reabsorvíveis nestes casos parecem colaborar na preservação da arquitetura óssea e gengival de regiões estéticas. Estudos mostram que quando estas técnicas são utilizadas, o nível da crista óssea ao redor de implantes, excelente parâmetro para mensurar a estabilidade periimplantar, torna-se mais estáveis a longo prazo ${ }^{16}$.

Visto a quantidade de critérios que influenciam o sucesso de implantes imediatos e a importância da boa conduta clínica para alcançar a alta taxa de sucesso da 
literatura, este trabalho tem por objetivo o relato de caso de instalação de implante imediato na região do dente 21 tomando os cuidados necessários para a manutenção das estruturas periodontais.

\section{RELATO DE CASO}

Paciente de 43 anos de idade compareceu ao curso de implantodontia da PGO (Pós-graduação em Odontologia) com queixa de dor provocada no dente $11 \mathrm{e}$ "bolinha" na gengiva (Figura 1).

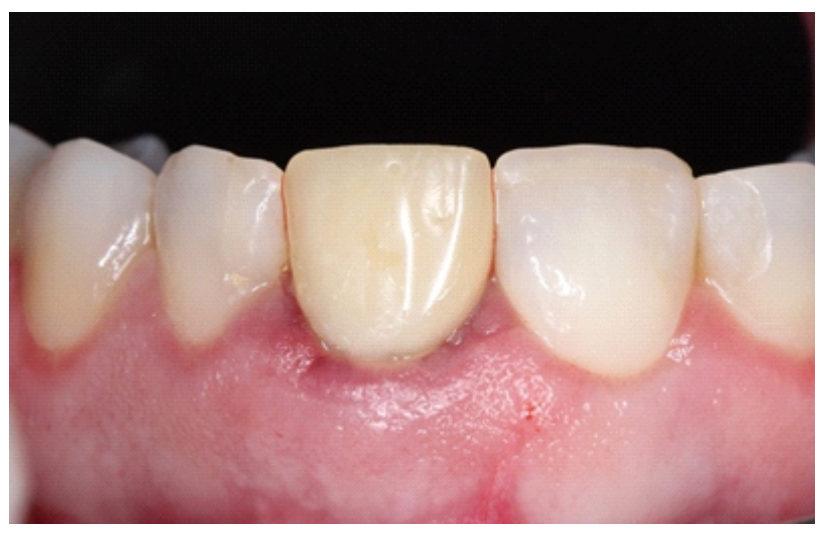

Figura 1 -Aspecto clínico do dente 21.

Após avaliação clínica e radiográfica foi observado núcleo metálico fundido, coroa metalocerâmica, conduto endodôntico parcialmente obturado e cárie radicular. Houve perda parcial da face vestibular do rebordo ósseo (Figura 2).

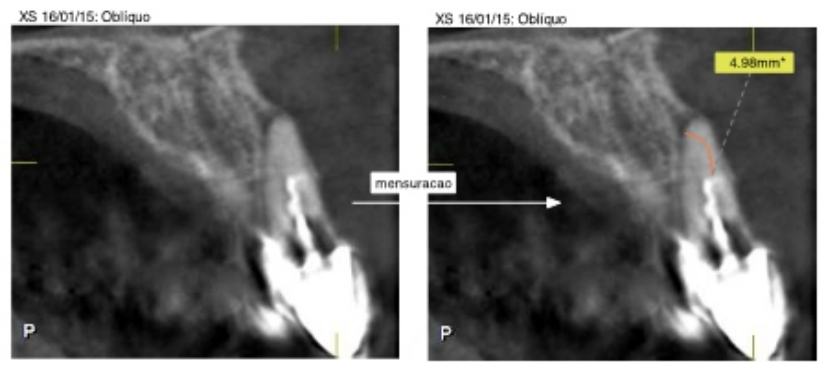

Figura 2 - Aspecto radiográfico do dente 21. Conduto endodôntico parcialmente obturado e cárie radicular.

Devido à baixa previsibilidade de sucesso na remoção do núcleo metálico e retratamento endodôntico, o planejamento do caso consistiu em exodontia atraumática do dente 11, instalação de implante imediato e confecção de coroa provisória com carga imediata. A paciente não possui desordens sistêmicas ou alterações nos exames laboratoriais (hemograma completo, glicemia em jejum e tempo de coagulação).
A antissepsia extra oral foi realizada com iodopolividona $10 \%$ (Riodeine ${ }^{\circledR}$, Rioquímica, São José do Rio Preto - SP, Brasil) e intra oral com digluconato de clorexidina $0.12 \%, 5 \mathrm{ml}$ por 1 minuto. A técnica anestésica utilizada foi infiltrativa com 3 tubetes de lidocaína HCL 2\% com epinefrina 1:100.000 (Alphacaine ${ }^{\circledR}$, Nova DFL, Taquara, Rio de Janeiro - RJ, Brasil). Em seguida foi feita incisão intrasulcular com bisturi lâmina 15 c (Advantive ${ }^{\circledR}$, Sterilance Medical, Suzhau - China), remoção na coroa metalocerâmica e luxação com periótomos e elevadores seldin 301. A exérese completa foi finalizada com fórceps no 69 e o alvéolo foi curetado com Cureta De Lucas (Figuras 3 e 4 ).

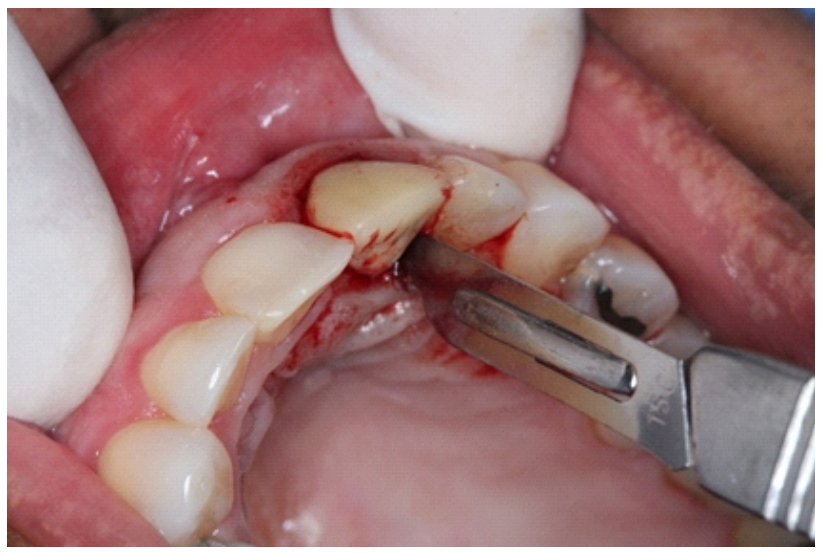

Figura 3 - Incisão intrasulcular e luxação com lâmina de bisturi 15c.

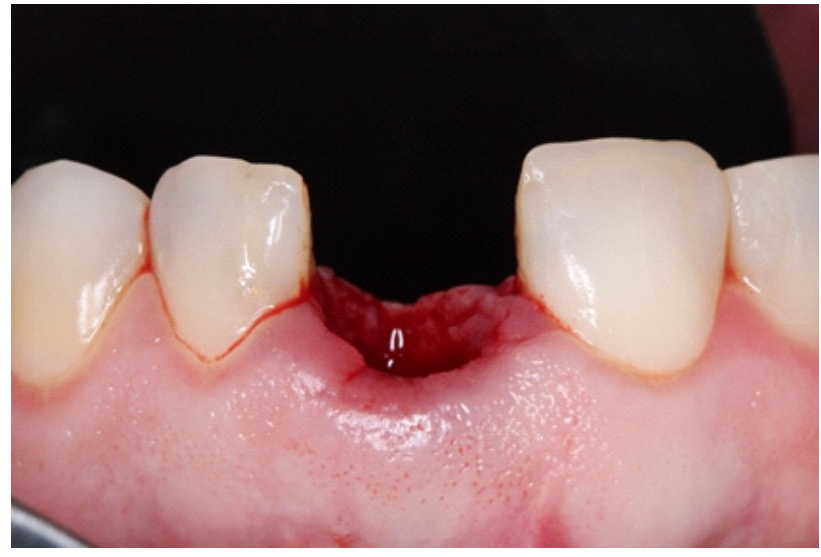

Figura 4 - Manutenção da integralidade dos tecidos periodontais após exodontia atraumática.

Após verificação das paredes do alvéolo com sonda milimetrada de ponta romba, o ponto de fresagem foi selecionado na parede palatina do alvéolo proporcionando posicionamento do implante dentário no cíngulo da futura coroa protética (Figura 5). Atentou-se também para o posicionamento mesio-distal, mantendo pelo menos $3 \mathrm{~mm}$ do implante da região do dente 22 e $1.5 \mathrm{~mm}$ do dente 11 (Figuras $6 \mathrm{e}$ 7). 


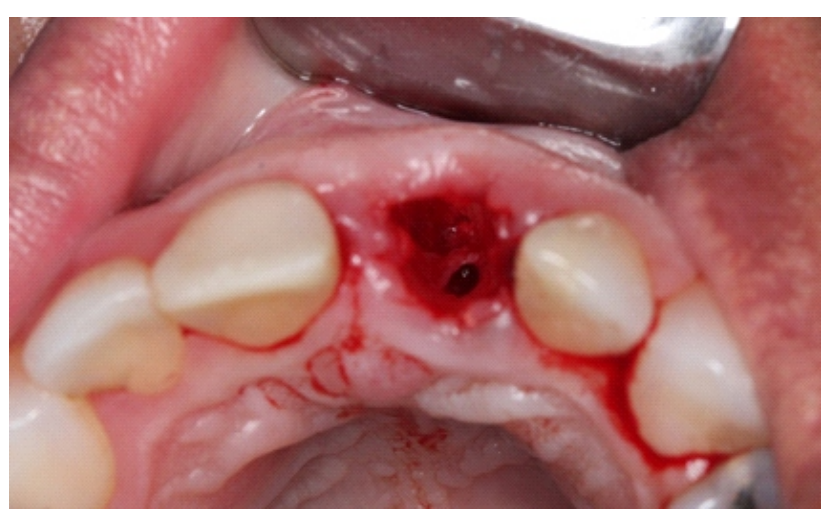

Figura 5 - Alvéolo cirúrgico localizado na parede palatina do alvéolo dentário.

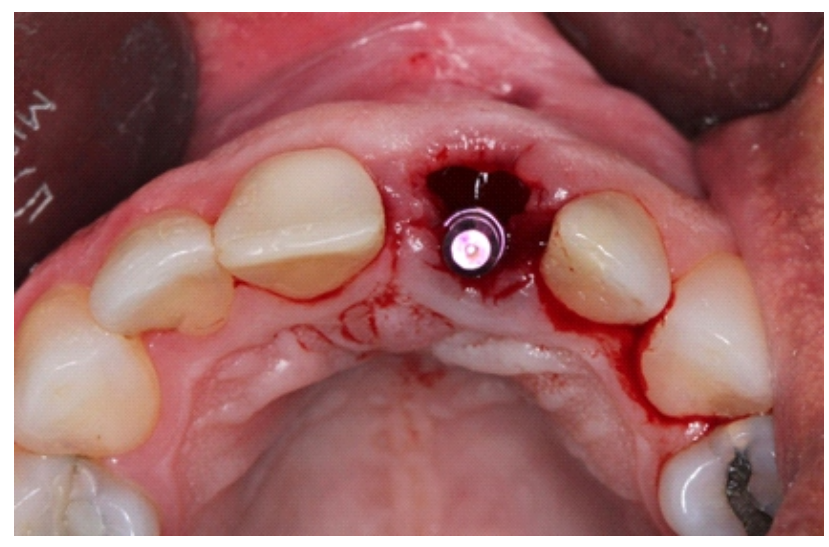

Figura 6 - Verificação do posicionamento antero-posterior da fresagem com paralelometro.

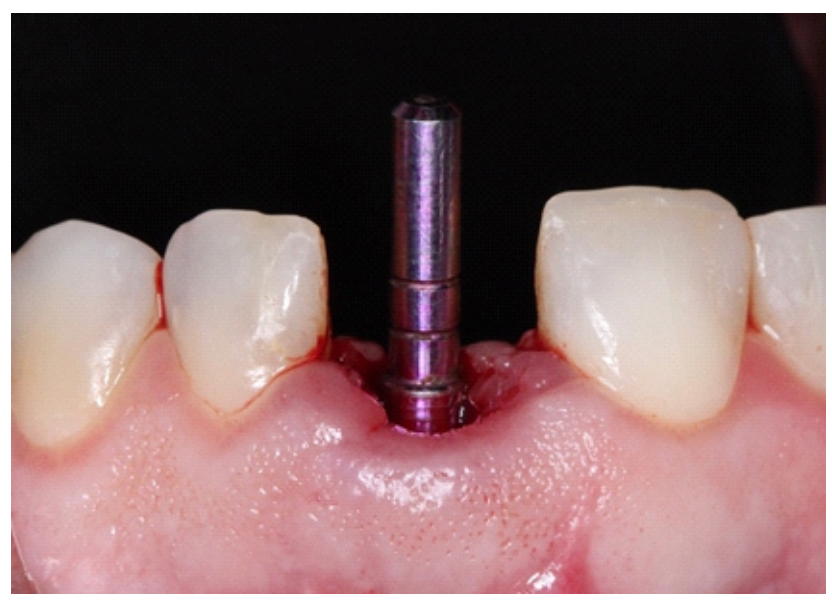

Figura 7 - Verificação do posicionamento maseio-distal da fresagem com paralelometro.

A fresagem foi realizada à $800 \mathrm{rpm}$, sob irrigação intensa de soro fisiológico 0,9\% (MedFlex ${ }^{\circledR}$, Eurofarma laboratórios S.A, São Paulo - SP, Brasil), utilizando motor cirúrgico elétrico (Driller ${ }^{\circledR}$, Carapicuíba - SP, Brasil) e seguindo a sequência: fresa lança, fresa $2 \mathrm{~mm}$ helicoidal, fresa $3.5 \mathrm{~mm}$ cônica (Sistema Neodent ${ }^{\circledR}$, Brasília - DF,
Brasil). O implante Cone Morse cilíndrici/cônico Avant (Systhex ${ }^{\circledR}$, Curitiba - PR, Brasil) de $13.0 \mathrm{~mm}$ foi instalado com torque de $40 \mathrm{~N}$ (Figuras 8 e 9).

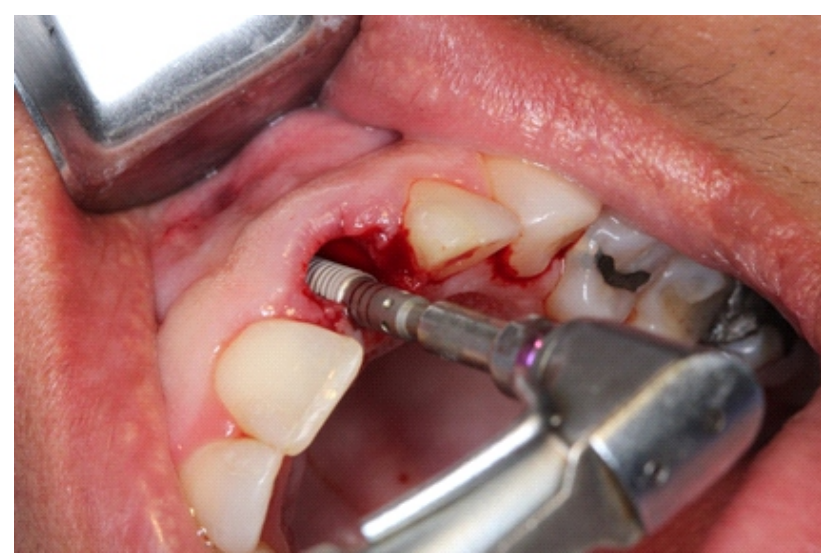

Figura 8 - Inserção do implante Avant com chave de contra ângulo a $30 \mathrm{rpm}$.

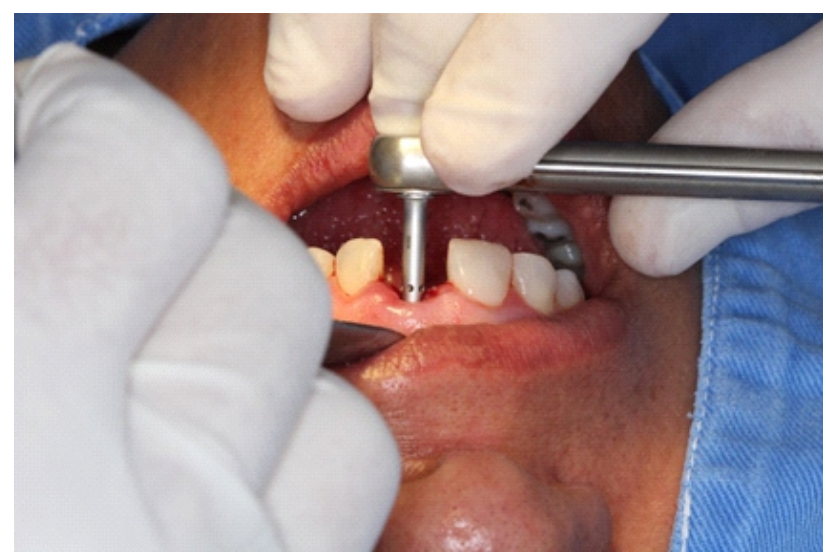

Figura 9 - Finalização da inserção do implante Avant com chave de catraca a $40 \mathrm{~N}$.

Após inserção do implante dentário o gap formado entre este e a parede vestibular do alvéolo foi preenchido com substituto ósseo sintético de hidroxiapatita (BlueBone ${ }^{\circledR}$, Regener, Curitiba - PR, Brasil) hidratado com soro fisiológico $0.9 \%$. Uma esponja hemostática (Hemospon®, Maquira Indústria de Produtos Odontológicos S.A, Maringá - PR, Brasil) foi posicionada sobre as partículas do enxerto para estabilização (Figuras 10 e 11). Não foram realizadas suturas. Um pilar Cone Morse de $2.5 \mathrm{~mm}$ (Neodent ${ }^{\circledR}$, Brasília - DF, Brasil) de altura e um cilindro provisório foi instalado para proporcionar confecção de provisório parafusado em resina acrílica. O provisório, após refinado acabamento e polimento, não comprimiu os tecidos periodontais e apresentou bom vedamento do alvéolo. Houve prescrição de nimesulida $100 \mathrm{mg}, 1$ comprimido a cada 12 horas por 2 dias e dipirona sódica $1 \mathrm{~g}$ a cada 6 horas por 2 dias. 


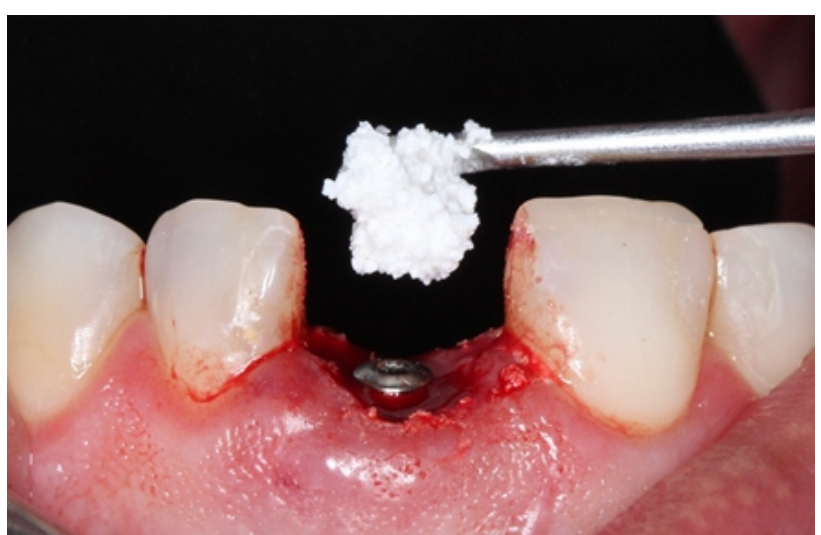

Figura 10 - Partículas de hidroxiapatita sintética hidratadas com soro fisiológico.

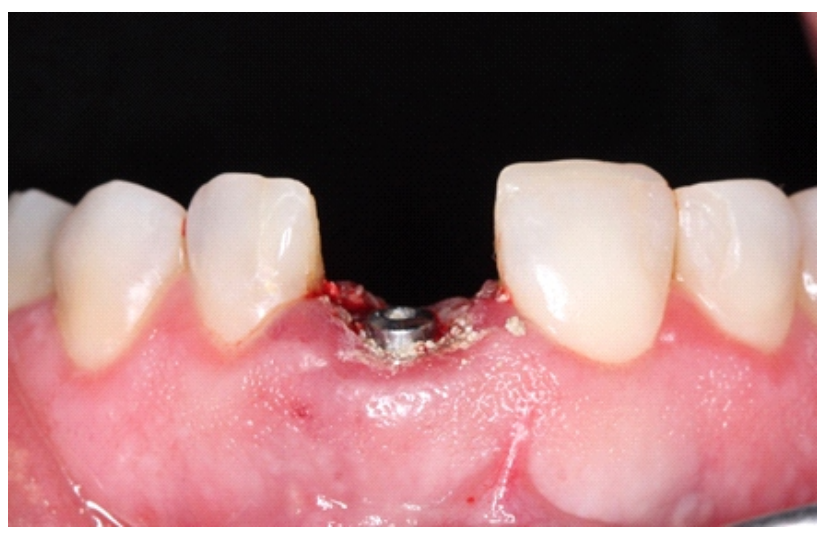

Figura 11 - Partículas de hidroxiapatita preenchendo gap entre parede vestibular do alvéolo e implante.

\section{DISCUSSÃO}

Durante o planejamento do caso, as opções de plano de tratamento foram: exodontia atraumática, regeneração óssea guiada com membrana não reabsorvível e confecção de provisório com instalação de implante tardio ou exodontia atraumática com instalação de implante imediato associado a regeneração óssea guiada e provisório imediato.

A segunda opção de tratamento foi a selecionada em conjunto com o paciente devido à grande previsibilidade da técnica e necessidade de menor número de consultas. $\mathrm{O}$ implante imediato, por diminuir uma etapa cirúrgica, oferece maior conforto ao paciente e otimiza o tempo de tratamento. Isso faz com que esta técnica seja a preferida em muitos $\operatorname{casos}^{17}$.

Após a seleção da técnica, a preocupação do clínico deve ser a correta prática de cada etapa cirúrgica. Durante a exodontia, o maior risco existente é a fratura da parede vestibular do alvéolo. Caso isto ocorra, aumentam as chances de reabsorção e criação de defeito anatômico e estético na vestibular. Para que isto seja evitado, o cirurgião deve realizar movimentos controlados evitando o movimento de alavanca horizontal. Aconselha-se o uso de periótomos em inserção no ligamento periodontal causando luxação dentária, seguida de movimento verticais de tração com fórceps.

Já a fresagem para instalação dos implantes osseointegrável é outro momento crítico da cirurgia, visto que o mal posicionamento das fresas acarretarão em malposicionamento do implante e componentes protéticos. É muito comum, nestes casos, a vestibularização dos implantes devido a anatomia dos alvéolos. O ideal é que os implantes sejam instalados na parede palatina dos alvéolos, induzindo o posicionamento da plataforma do implante do cíngulo protético da região.

A terceira etapa crítica do procedimento pode ser considerada o preenchimento do gap existente entre superfície do implante instalado e a parede vestibular do alvéolo fresco. O preenchimento apenas com coágulo, dependendo da espessura da parede vestibular, pode não ser suficiente para a osteogênese originando defeito óssea. $\mathrm{O}$ preconizado pela literatura atualmente é o preenchimento do gap com substitutos ósseos de lenta reabsorção, que servirão de arcabouço para proliferação celular. A utilização de membranas é facultativa, podendo ser utilizado também esponjas de fibrinas apenas para estabilização das partículas ${ }^{18}$.

É necessário também a boa seleção do componente protético, bem como a boa confecção do provisório imediato, o qual deve apresentar excelente polimento, bom perfil de emergência, bom selamento do alvéolo e ausência de contatos oclusais exagerados. Estes critérios são fundamentais para o sucesso do implante imediato instalado seja atingido ${ }^{19}$.

\section{CONCLUSÃO}

Conclui-se que a instalação de implantes imediatos, quando realizada com segurança e respeitando os princípios biológicos e técnicos, é uma boa opção para preservação da arquitetura periodontais em regiões estéticas.

\section{REFERÊNCIAS}

1. Kirsten WS, Laurens H, Nicolaas AB, Arjan V, Henny JAM, Gerry MR. Immediate placement of dental implants in the esthetic zone: a systematic review and pooled analysis. J Periodontol. 2014;85(7):241-50.

2. Testori T, Weinstein T, Scutellà F, Wang HL, Zucchelli G. Implant placement in the esthetic area: criteria for positioning single and 
multiple implants. Periodontol 2000. 2018;77(1):176-96.

3. Kan JYK, Rungcharassaeng K, Deflorian M, Weinstein T, Wang HL, Testori T. Immediate implant placement and provisionalization of maxillary anterior single implants. Periodontol 2000. 2018;77(1):197-212.

4. Kan JYK, Roe P, Rungcharassaeng K, Patel R, Waki T, Lozada JL, et al. Classification of sagittal root position in relation to the anterior maxillary osseous hous-ing for immediate implant placement: a cone beam com- puted tomography study. Int J Oral Maxillofac Implants. 2011;26(4):873-6.

5. Kan JYK, Rungcharassaeng K. Immediate placement and provisionalization of maxillary anterior single implant: a surgical and prosthodontic rationale. Pract Periodontics Aesthet Dent. 2000;12(9):817-24.

6. Palattella P, Torsello F, Cordaro L. Two-year prospective clinical comparison of immediate replacement vs. immediate restoration of single tooth in the esthetic zone. Clin Oral Implants Res. 2008;19(11):1148-53.

7. Shibly O, Patel N, Albandar JM, Kutkut A. Bone regeneration around implants in periodontally compromised patients: a randomized clinical trial of the effect of immediate implant with immediate loading. J Periodontol. 2010;81(12):1743-51.

8. Esposito M, Grusovin MG, Polyzos IP, Felice P, Worthington HV. Interventions for replacing missing teeth: dental implants in fresh extraction sockets (immediate, immediate-delayed and delayed implants). Cochrane Database Syst Rev. 2010;8(9):9-12.

9. Lee J, Park D, Koo KT, Seol YJ, Lee YM. Comparison of immediate implant placement in infected and non-infected extraction sockets: a systematic review and meta-analysis. Acta Odontol Scand. 2018;76(5):338-45.

10. Canellas JVDS, Medeiros PJD, Figueredo CMDS, Fischer RG, Ritto FG. Which is the best choice after tooth extraction, immediate implant placement or delayed placement with alveolar ridge preservation? A systematic review and meta-analysis. J Craniomaxillofac Surg. 2019;47(11):1793-802.

11. Yan Q, Xiao LQ, Su MY, Mei Y, Shi B. Soft and hard tissue changes following immediate placement or immediate restoration of singletooth implants in the esthetic zone: a systematic review and metaanalysis. Int J Oral Maxillofac Implants. 2016;31(6):1327-40.

12. Hur Y, Ogata Y. No clinically significant differences in crestal bone loss between immediate implant placement and implants placed in healed bone: a systematic review of articles published from 1966 through 2012. JAm Dent Assoc. 2016;147(12):987-9.

13. Tettamanti L, Andrisani C, Bassi MA, Vinci R, Silvestre-Rangil J, Tagliabue A. Post extractive implant: evaluation of the critical aspects. Oral Implantol (Rome). 2017;10(2):119-28.

14. Mello CC, Lemos CAA, Verri FR, Dos Santos DM, Goiato MC, Pellizzer E.P. Immediate implant placement into fresh extraction sockets versus delayed implants into healed sockets: a systematic review and meta-analysis. Int J Oral Maxillofac Surg. 2017;46(9):1162-77.

15. Blanco J, Carral C, Argibay O, Liñares A. Implant placement in fresh extraction sockets. Periodontol 2000. 2019;79(1):151-67.

16. Araújo MG, Silva CO, Souza AB, Sukekava F. Socket healing with and without immediate implant placement. Periodontol 2000. 2019;79(1):168-77.
17. Kan JYK, Rungcharassaeng K, Deflorian M, Weinstein T, Wang HL, Testori T. Immediate implant placement and provisionalization of maxillary anterior single implants. Periodontol 2000. 2018;77(1):197-212.

18. Santos PL, Gulinelli JL, Telles CS, Betoni Júnior W, Okamoto R, Chiacchio Buchignani $\mathrm{V}$, et al. Bone substitutes for peri-implant defects of postextraction implants. Int J Biomater. 2013;(3):1-7.

19. Van Nimwegen WG, Raghoebar GM, Zuiderveld EG, Jung RE, Meijer HJA, Mühlemann S. Immediate placement and provisionalization of implants in the aesthetic zone with or without a connective tissue graft: A 1-year randomized controlled trial and volumetric study. Clin Oral Implants Res. 2018;29(7):671-8. 